\title{
Differential activation of c-Fos in the paraventricular nuclei of the hypothalamus and thalamus following myocardial infarction in rats
}

\author{
HYUN-JIN TAE ${ }^{1,2^{*}}$, SEUNG MIN PARK ${ }^{3,4^{*}}$, JEONG HWI CHO ${ }^{5}$, IN HYE KIM ${ }^{5}$, JI HYEON AHN ${ }^{1}$, \\ JOON HA PARK ${ }^{5}$, MOO-HO WON ${ }^{5}$, BAI HUI CHEN ${ }^{6}$, BICH-NA SHIN ${ }^{6}$, MYOUNG CHEOL SHIN ${ }^{4}$, \\ CHOONG HYUN LEE ${ }^{7}$, SEONGKWEON HONG $^{8}$, JAE-CHUL LEE $^{5}$ and JUN HWI CHO ${ }^{4}$
}

${ }^{1}$ Department of Biomedical Science and Research Institute for Bioscience and Biotechnology, Hallym University,

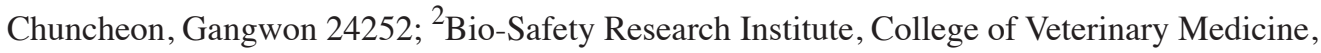

Chonbuk National University, Iksan, Jeollabuk-do 54596; ${ }^{3}$ Department of Emergency Medicine, Sacred Heart Hospital, College of Medicine, Hallym University, Anyang, Gyeonggi 14068; Departments of ${ }^{4}$ Emergency Medicine and ${ }^{5}$ Neurobiology, School of Medicine, Kangwon National University, Chuncheon, Gangwon 24341; ${ }^{6}$ Department of Physiology, College of Medicine, Hallym University, Chuncheon, Gangwon 24252; ${ }^{7}$ Department of Pharmacy, College of Pharmacy, Dankook University, Cheonan, Chungcheongnam 16890;

${ }^{8}$ Department of Surgery, School of Medicine, Kangwon National University, Chuncheon, Gangwon 24341, Republic of Korea

Received August 16, 2015; Accepted August 26, 2016

DOI: $10.3892 / \mathrm{mmr} .2016 .5731$

\begin{abstract}
Proto-oncogene c-Fos (c-Fos) is frequently used to detect a pathogenesis in central nervous system disorders. The present study examined changes in the immunoreactivity of c-Fos in the paraventricular nucleus of the hypothalamus (PVNH) and paraventricular nucleus of the thalamus (PVNT) following myocardial infarction (MI) in rats. Infarction in the left ventricle was examined by Masson's trichrome staining. Neuronal degeneration was monitored for 56 days after MI using crystal violet and Fluoro-Jade B histofluorescence staining. Changes in the immunoreactivity of c-Fos were determined using immunohistochemistry for c-Fos. The average infarct size of the left ventricle circumference was $\sim 44 \%$ subsequent to MI. Neuronal degeneration was not detected in PVNH and PVNT following MI. c-Fos immunoreactive $\left(^{+}\right)$cells
\end{abstract}

Correspondence to: $\mathrm{Dr}$ Jae-Chul Lee, Department of Neurobiology, School of Medicine, Kangwon National University, 1 Kangwondaehak-gil, Chuncheon, Gangwon 24341, Republic of Korea

E-mail: anajclee@kangwon.ac.kr

Professor Jun Hwi Cho, Department of Emergency Medicine, School of Medicine, Kangwon National University, 156 Baengnyeong-ro, Chuncheon, Gangwon 24341, Republic of Korea

E-mail: cjhmd@kangwon.ac.kr

*Contributed equally

Key words: myocardial infarction, c-Fos, rat, paraventricular nucleus of the hypothalamus, paraventricular nucleus of the thalamus were infrequently observed in the nuclei of the sham-group. However, the number of $\mathrm{c}-F o s^{+}$cells was increased in the nuclei following $\mathrm{MI}$ and peaked in the PVNH and PVNT at 3 and 14 days, respectively. The number of $\mathrm{c}-F o s^{+}$cells were comparable with the sham group at 56 days after MI. Therefore, MI may induce c-Fos immunoreactivity in PVNH and PVNT, this increase of c-Fos expression levels may be associated with the stress that occurs in the brain following MI.

\section{Introduction}

Myocardial infarction (MI) inhibits blood flow to the heart and damages cardiac muscle, which may lead to heart failure, an irregular heartbeat or cardiac arrest (1). MI is one of the predominant global diseases due to its high mortality and morbidity rate, it is also a burden on the healthcare system in various countries (2).

The communication between the brain and the heart is considered to be bidirectional (3-5). During cardiac disease, the brain signals to the heart; cardiac dysfunction initially occurs and then the brain provides feedback to the heart via sympathetic drive or fluid regulation to sustain the cardiac disease state $(2,6)$.

Previous studies have examined the central structures or brain neurons associated with MI and heart failure (7-11). Monitoring of proto-oncogene c-Fos (c-Fos) expression levels has been established as a reliable technique to identify neural populations of metabolically activated brain regions $(12,13)$. The selective expression of c-Fos has previously been observed in the rat brain following heart failure (11). It is generally accepted that neuronal activation or changes in the brain occur following MI, however, the mechanisms involved remain poorly understood. The paraventricular nucleus of the 
hypothalamus (PVNH) and the paraventricular nucleus of the thalamus (PVNT), which exhibit c-Fos expression following stresses, including capsaicin-induced nociceptive pain in rats (14). However, to the best of our knowledge, this effect has not been reported during MI. Therefore, the present study examined the changes in c-Fos expression levels in the PVNH and PVNT of rats to understand the pathophysiology and improve the management of MI $(15,16)$.

\section{Materials and methods}

Induction of MI. Male Sprague-Dawley rats (12 weeks of age; body weight, 300-320 g) were obtained from the Experimental Animal Center at Kangwon National University (Chuncheon, South Korea). A total of 98 male rats were housed in individual cages (temperature, $23^{\circ} \mathrm{C}$; humidity, 60\%) under a $12 \mathrm{~h}$ light/dark cycle, and provided with commercial chow and water ad libitum and throughout the experimental period. The procedures for animal handling and care were in compliance with the Guide for the Care and Use of Laboratory Animals, and were approved by the Institutional Animal Care and Use Committee at Kangwon National University (Chuncheon, South Korea).

MI was induced as described in our previous study (17). Briefly, the animals were intubated and ventilated with a small animal ventilator (model SAR-830/P; CWE, Inc., Ardmore, PA, USA). The left coronary artery was permanently ligated below the left atrial appendage. Sham-operated animals were subjected to the same surgical procedures without ligation of the left coronary artery.

Tissue processing for histology. The rats were anesthetized with intraperitoneal injection of pentobarbital sodium (30 mg/kg; JW Pharmaceutical, Seoul, Korea) at 1, 3, 7, 14, 28 and 56 days ( $n=7$ at each time point) after MI induction. Subsequently, they were perfused via the abdominal aorta with $0.1 \mathrm{M}$ phosphate-buffered saline (PBS; pH 7.4) followed by $4 \%$ paraformaldehyde in $0.1 \mathrm{M}$ phosphate-buffer ( $\mathrm{pH} 7.4$ ). The hearts were excised and embedded in paraffin blocks and sectioned into $6 \mu \mathrm{m}$ sections at $600 \mu \mathrm{m}$ intervals. The brains were removed and embedded in tissue-freezing medium and serially sectioned into $30 \mu \mathrm{m}$ coronal sections using a cryostat (Leica Microsystems GmbH, Wetzlar, Germany).

Masson's trichrome staining. The heart sections were stained to examine the histology of the heart using Masson's trichrome staining as previously described by Ahmet et al (15) the sections were placed in the Biebrich scarlet-acid fuchsin solution (Sigma-Aldrich; Merck Millipore, Darmstadt, Germany) and aniline blue (Sigma-Aldrich; Merck Millipore) to detect the area of infarction in the heart tissue of MI-induced rats. Images were obtained using a light microscope (BX53, Olympus, Hamburg, Germany) equipped with a digital camera (DP72, Olympus) connected to a PC monitor. Whole images of the heart were merged by image analyzing system Optimas version 6.5 (CyberMetrics, Phoenix, AZ, USA).

Crystal violet (CV) and Fluoro-Jade B (F-JB) histofluorescence staining. To examine neuronal damage in the diencephalon following MI, CV staining and F-J B histofluorescence staining were performed as previously described (18). In brief, the sections were stained with $1.0 \%$ (w/v) CV acetate (Sigma-Aldrich; Merck Millipore) and dehydrated. For F-J $\mathrm{B}$ histofluorescence, the sections were immersed in $0.0004 \%$ F-J B (Histo-Chem, Inc., Jefferson, AR, USA) staining solution. The sections were washed with 0.1 M PBS and examined using an epifluorescent microscope (Zeiss GmbH, Göttingen, Germany) with blue (450-490 nm) excitation light and a barrier filter. Five randomly selected microscope fields (x400 magnification) were photographed to represent each rat.

Immunohistochemistry for c-Fos. c-Fos immunohistochemistry in the diencephalon was performed according to our previous study (18). Briefly, the sections were incubated with primary rabbit anti-c-Fos (cat. no. . sc-52; 1:200; Santa Cruz Biotechnology, Inc., Dallas, TX, USA), followed with secondary antibody incubation at room temperature for $2 \mathrm{~h}$ (cat. no. BA1000; 1:200; Vector Laboratories, Inc., Burlingame, CA, USA) and developed using a Vectastain ABC kit (Vector Laboratories, Inc.). They were visualized with 3,3'-diaminobenzidine in $0.1 \mathrm{M}$ Tris- $\mathrm{HCl}$ buffer. Five randomly selected digital images of the PVNH and PVNT groups per rat were captured using Olympus light microscope equipped with a digital camera (DP72, Olympus) connected to a PC monitor

Western blot analysis. To obtain the accurate data for changes in level of c-Fos protein in the PVNH and PVNT following $\mathrm{MI}$, the animals ( $\mathrm{n}=7$ at each time point) were sacrificed at 1, 3, 7, 14, 28 and 56 day following the induction of MI and the PVNH and PVNT tissue used for western blot analysis. As previously described (19), the brain was transversely cut into $400-\mu \mathrm{m}$ thick sections on a vibratome (Leica Microsystems GmbH). The PVNH and the PVNT were dissected with a surgical blade under stereoscopic microscope. The tissues were homogenized in $50 \mathrm{mM}$ PBS (pH 7.4) containing $0.1 \mathrm{mM}$ ethylene glycol bis (2-aminoethyl ether)-N,N,N',N' tetraacetic acid ( $\mathrm{pH} 8.0$ ), $0.2 \%$ Nonidet P-40, $10 \mathrm{mM}$ ethylendiamine tetraacetic acid ( $\mathrm{pH} 8.0), 15 \mathrm{mM}$ sodium pyrophosphate, $100 \mathrm{mM} \beta$-glycerophosphate, $50 \mathrm{mM}$ $\mathrm{NaF}, 150 \mathrm{mM} \mathrm{NaCl}, 2 \mathrm{mM}$ sodium orthovanadate, $1 \mathrm{mM}$ phenylmethylsulfonyl fluoride and $1 \mathrm{mM}$ dithiothreitol (DTT). Following centrifugation at in $16,000 \mathrm{x}$ g for $20 \mathrm{~min}$ at $4^{\circ} \mathrm{C}$, the protein level in the supernatant was determined using a Micro BCA protein assay kit with bovine serum albumin as the standard (Pierce; Thermo Fisher Scientific, Inc., Waltham, MA, USA). Aliquots containing $20 \mu \mathrm{g}$ total protein were boiled in loading buffer containing $150 \mathrm{mM}$ Tris $(\mathrm{pH} \mathrm{6.8)}$, 3 mM DTT, $6 \%$ sodium dodecyl sulfate, $0.3 \%$ bromophenol blue and $30 \%$ glycerol. Subsequently, each aliquot was loaded onto a $12.5 \%$ polyacrylamide gel for electrophoresis. The gels were then transferred to nitrocellulose transfer membranes (Pall Corporation, East Hills, NY, USA). To reduce background staining, the membranes were incubated with $5 \%$ non-fat dry milk in PBS containing $0.1 \%$ Tween 20 for 45 min and then with rabbit anti-c-Fos (cat. no. . sc-52; 1:1,500; Santa Cruz Biotechnology, Inc.) for $24 \mathrm{~h}$ at $4^{\circ} \mathrm{C}$, followed by incubation at room temperature for $1 \mathrm{~h}$ with peroxidase-conjugated goat anti-rabbit IgG (cat. no. A0545; 1:200; Sigma-Aldrich; Merck Millipore) and an enhanced chemiluminescence kit (Pierce; Thermo Fisher Scientific, Inc.) was used for detection. 
Loading controls were performed using $\beta$-actin incubated at $4^{\circ} \mathrm{C}$ overnight (cat. no. ab8227; 1:5,000; Abcam, Cambridge, MA, USA). Densitometric analysis for the quantification of the bands was performed using ImageJ version 1.46 software (National Institutes of Health, Bethesda, MD, USA), which was used to determine the relative optical density (ROD). The levels of c-Fos were normalized to the level of $\beta$-actin. A ratio of the ROD was calibrated as the percentage, with the PM 3 group designated as $100 \%$

Data analysis. The size of the MI induced was calculated using the method previously described by Ahmet et al (15) as an average percentage of the left ventricular endocardial and epicardial circumferences that were identified as the infarcted area in the Masson's trichrome-stained tissues. The mean number of c-Fos-immunoreactive $\left(^{+}\right)$cells was counted in a $250 \times 250 \mu \mathrm{m}$ square in five randomly selected images of PVNH and PVNT in each rat. Cell counts were obtained by averaging the total number of cells from each animal per group.

Statistical analysis. Data are presented as the mean \pm standard error. One-way analysis of variance and Tukey's post-hoc test were used in order to compare the difference of c-Fos immunoreactivity and protein levels between sham- and MI-operated rat groups. SAS software version 9.2 (SAS Institute Inc., Cary, NC, USA) was used to perform the statistical analyses. $\mathrm{P}<0.05$ was considered to indicate a statistically significant difference.

\section{Results}

MI induction. Masson's trichrome staining determined that the infarcted region encompassed between 41 and $46 \%$ of the ventricular circumference of the left ventricle following MI (Fig. 1). No difference in the infarcted area was observed between the different time-point groups. Viable myocardium was stained red and fibrosis caused by infarction damage was stained blue (Fig. 1). The sham-operated group was used as a control group and no blue staining was observed. By contrast, the infarcted zone in the MI-operated group had pronounced blue scar tissue. The infarct wall of the left ventricle was thinner (indicated with arrows in Fig. 1) following MI, whereas the non-infarct wall exhibited hypertrophy (Fig. 1B and C). Collagen and myofibroblasts accumulated in the infarct wall over time and replaced with necrotic myocardium by 56 days after MI (Fig. 1D).

MI-induced neuronal damage. The PVNH and PVNT were identified by $\mathrm{CV}$ staining (Fig. 2). The staining pattern observed in the MI-operated group was comparable with the sham group (Fig. 2B, C, H and I). F-J B fluorescence staining was used to detect neurodegenerating structures and F-J B ${ }^{+}$ cells were not detected in the PVNH (Fig. 2D-F) and PVNT (Fig. 2J-L) of the sham- or MI-operated groups.

c-Fos immunoreactivity following MI. In the sham-operated group, c-Fos immunoreactivity was infrequently observed in the PVNH and PVNT (Fig. 3). By contrast, the number of

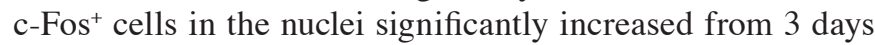

onwards following coronary artery ligation compared with the sham group $(\mathrm{P}<0.05$; Figs. 3 and $4 \mathrm{~A})$. In the $\mathrm{PVNH}$, the mean number of $\mathrm{c}^{-\mathrm{Fos}^{+}}{ }^{+}$cells peaked at 3 days after MI $\left(140.5 \pm 7.2 / \mathrm{mm}^{2}\right.$ per section; Figs. $3 \mathrm{~B}$ and 4$)$. c-Fos ${ }^{+}$cells subsequently decreased over time (Figs. 3C-F and 4). In the PVNT, c-Fos ${ }^{+}$cells were gradually increased and their mean number was significantly greater at 3 days after MI compared with the sham group $(\mathrm{P}<0.05$; Fig. $4 \mathrm{~B})$ and peaked at 14 days after MI (159.7 $\pm 16.4 / \mathrm{mm}^{2}$ per section; Figs. 3H-L and 4B). c-Fos ${ }^{+}$cells were infrequently observed in PVNH and PVNT 56 days after MI (Figs. 3F, L and 4).

c-Fos protein expression levels increase following MI. Western blot analysis determined that the changes in c-Fos protein expression levels in PVNH and PVNT following coronary artery ligation (Fig. 5) were similar to the immunohistochemistry findings. c-Fos protein expression levels in the PVNH peaked at the 3 days after MI, and were significantly increased at 3, 7 and 14 days compared with the sham group $(\mathrm{P}<0.05$; Fig. 5B). The protein expression levels subsequently decreased gradually over time following MI. In the PVNT, c-Fos protein expression levels were significantly increased at 7 days after $\mathrm{MI}$ compared with the sham group $(\mathrm{P}<0.05$; Fig. 5C) and peaked at 14 days, a decrease in c-Fos expression was subsequently was observed. The c-Fos protein levels in the group observed 56 days after MI were comparable with the sham-operated group (Fig. 5).

\section{Discussion}

c-Fos is a good biological marker for detecting pathogenesis in the central nervous system. Few studies regarding change in MI-induced c-Fos in the brain have been reported $(11,20)$, and the change of c-Fos in the PVNH and PVNT following MI remains to be elucidated. The average infarct size was $\sim 4 \%$ of the left ventricle circumference following MI. Neuronal damage was not detected in PVNH and PVNT following MI. c-Fos ${ }^{+}$ cells were not detected in the sham-group. However, the number of c-Fos ${ }^{+}$cells in the PVNH and PVNT was increased following the induction of $\mathrm{MI}$ and peaked at 3 and 14 days, respectively. c-Fos ${ }^{+}$cells were not detected in the PVNH and PVNT at 56 days after MI induction. Therefore, MI significantly induces c-Fos immunoreactivity and increases c-Fos protein expression levels in the PVNH and PVNT. The increase of c-Fos expression levels may be associated with increased cerebral stress that occurs following MI.

Limiting the blood supply to the left anterior descending coronary artery has been previously used to induce MI in experimental animals, which may model human heart failure (21). Infarcts of similar magnitude have been reported to be associated with reduced cardiac function $(15,22)$. The present study did not identify a significant difference between the size of the infarcts at any time following the induction of MI.

Previous studies have demonstrated that MI induces neuronal damage in several regions of the brain, including the hippocampal CA1 region and amygdala (5,23-25). However, neuronal damage was not detected in the PVNH and PVNT using CV and F-J B histofluorescence staining in the present study. Therefore, it is possible that neurons in the PVNH and PVNT may be less susceptible to MI-induced damage. 
A

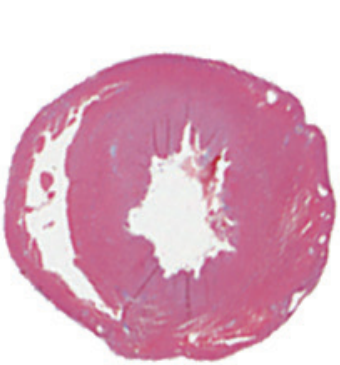

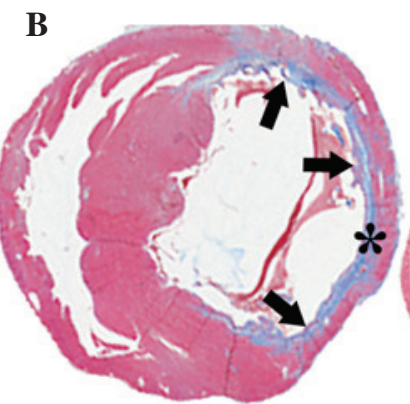

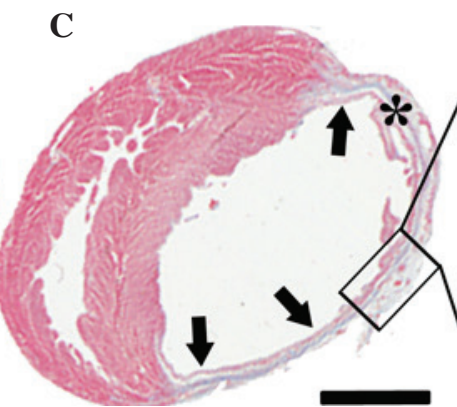

D

Figure 1. Masson's trichrome staining in the (A) sham- and MI-operated rats at (B) 7 and (C) 56 days after coronary artery ligation. Scale bar, $5 \mathrm{~mm}$. The arrows indicate that the infarct wall of the left ventricle becomes thinned following MI, whereas hypertrophy is evident in the non-infarct wall. Collagen (asterisks) was observed in the infarct wall of the LV 3 days after MI and continued to accumulate with time. (D) At 56 days after MI, Masson's trichrome staining demonstrated that necrotic myocardium was replaced with collagen (asterisks) and myofibroblasts. Scale bar, $50 \mu \mathrm{m}$ in (D). MI, myocardial infarction; $\mathrm{LV}$, left ventricle.
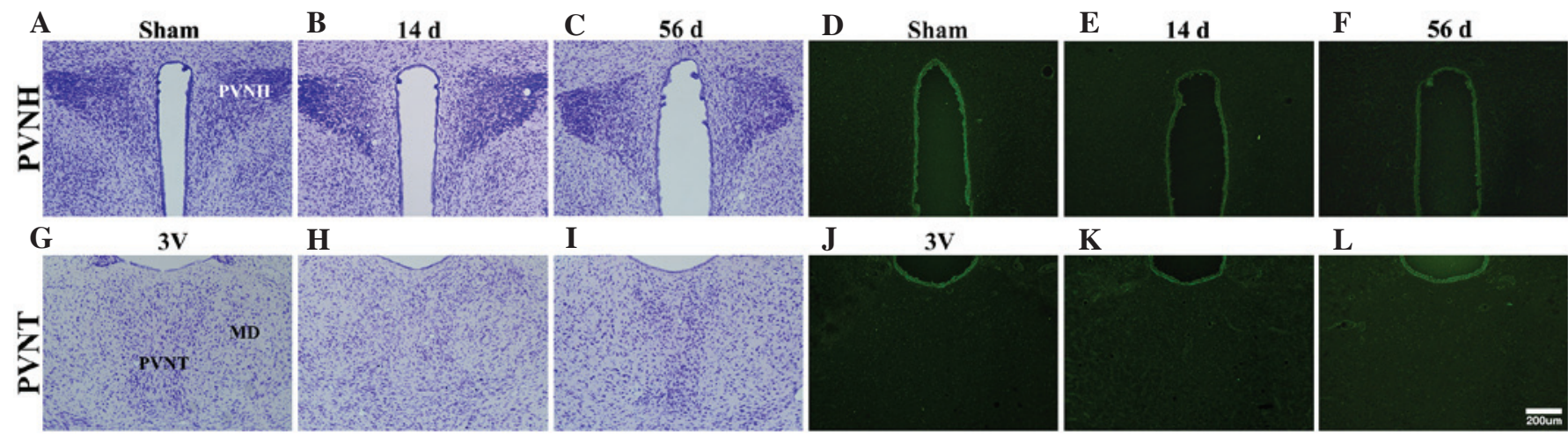

L
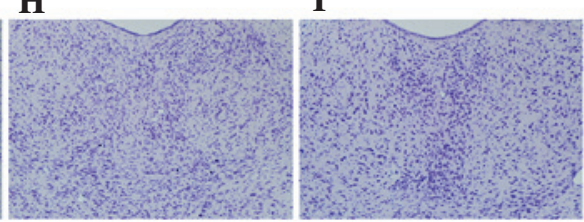

Figure 2. Crystal violet and F-J B histofluorescence staining of the PVNH and PVNT. Crystal violet staining of PVNH in (A) sham, and (B) $14 \mathrm{~d}$ and (C) $56 \mathrm{~d}$ myocardial infarction-operated groups. F-J B histofluorescence of PVNH in (D) sham, and (E) $14 \mathrm{~d}$ and (F) $56 \mathrm{~d}$ myocardial infarction-operated groups. Crystal violet staining of PVNT in (G) sham, and (H) $14 \mathrm{~d}$ and (I) $56 \mathrm{~d}$ myocardial infarction-operated groups. F-J B histofluorescence of PVNT in (J) sham, and (K) $14 \mathrm{~d}$ and (L) $56 \mathrm{~d}$ myocardial infarction-operated groups. F-J B ${ }^{+}$cells were not found in all groups. Scale bar, $200 \mu \mathrm{m}$. d, day; PVNH, paraventricular nucleus of the hypothalamus; PVNT, paraventricular nucleus of the thalamus; F-J B, Fluoro-Jade B; 3V, 3rd ventricle; MD, mediodorsal thalamic nucleus.

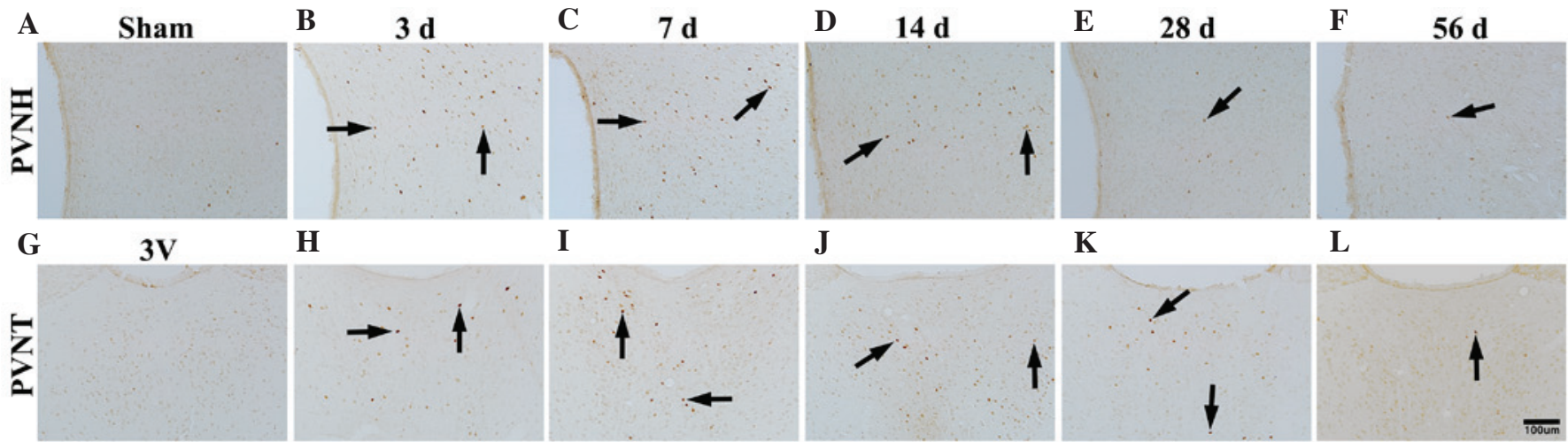

Figure 3. c-Fos immunohistochemistry in the PVNH and PVNT. Staining was performed in the PVNH of (A) sham and MI-operated groups at (B) $3 \mathrm{~d}$, (C) $7 \mathrm{~d}$, (D) $14 \mathrm{~d}$, (E) $28 \mathrm{~d}$ and (F) $56 \mathrm{~d}$. Staining was performed in the PVNT of (G) sham and MI-operated groups at (H) $3 \mathrm{~d}$, (I) $7 \mathrm{~d}$, (J) $14 \mathrm{~d}$, (K) $28 \mathrm{~d}$ and (L) $56 \mathrm{~d}$. In the sham-operated group, c-Fos immunoreactivity was infrequently detected. The arrows indicate the c-Fos immunoreactive cells, the number of c-Fos immunoreactive cells peaked in the PVNH and PVNT at 3 and 14 days, respectively, subsequent to MI. Scale bar, $100 \mu \mathrm{m}$. d, day; PVNH, paraventricular nucleus of the hypothalamus; PVNT, paraventricular nucleus of the thalamus; MI, myocardial infarction; c-Fos, proto-oncogene c-Fos.

The PVNH contains sub-populations of neurons that may be activated by various stresses and physiological changes (26). Additionally, it is the primary site for the activation of vasopressin-synthesizing neurons in humans and rats following MI (3). Therefore, the PVNH may be involved in altering volume reflex, which has been previously observed in MI (11).
The PVNT is also important for the arousal/attention response and modulating the process of stress-associated information following MI (27). PVNT is activated following various stresses, including immobilization, pain and fear (28-31).

c-Fos is associated with the neuronal activation underlying learning and memory processes in the central nervous system 
A

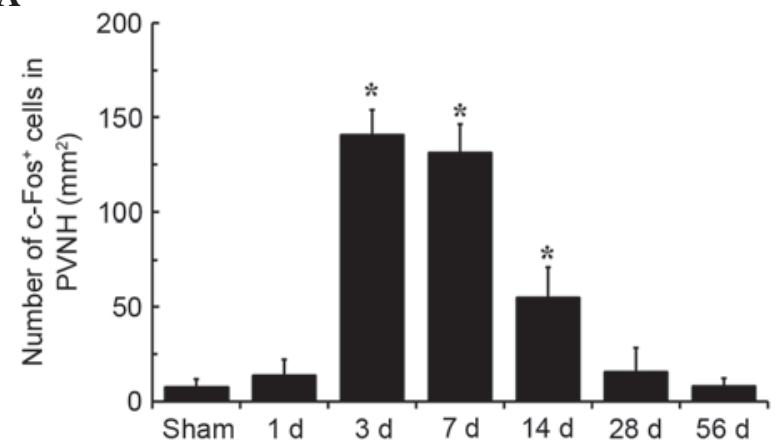

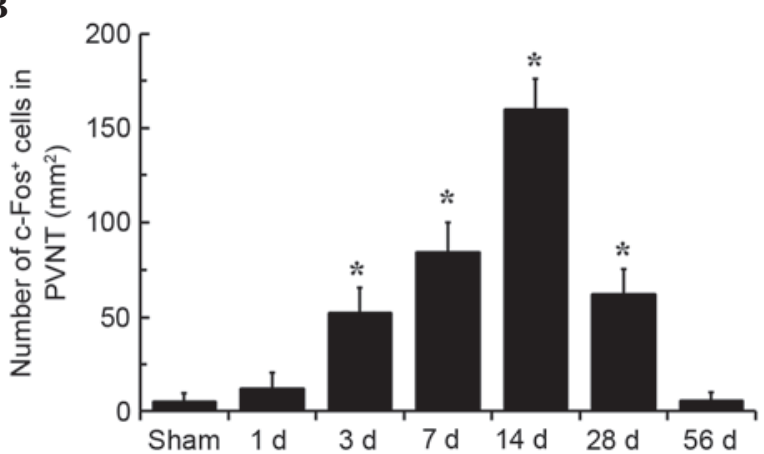

Figure 4. Mean number of c-Fos immunoreactive cells in the (A) PVNH and (B) PVNT (n=7 per group). "P<0.05 vs. sham group. Data are presented as the mean \pm standard error. c-Fos, proto-oncogene c-Fos; PVNH, paraventricular nucleus of the hypothalamus; d, day; PVNT, paraventricular nucleus of the thalamus.
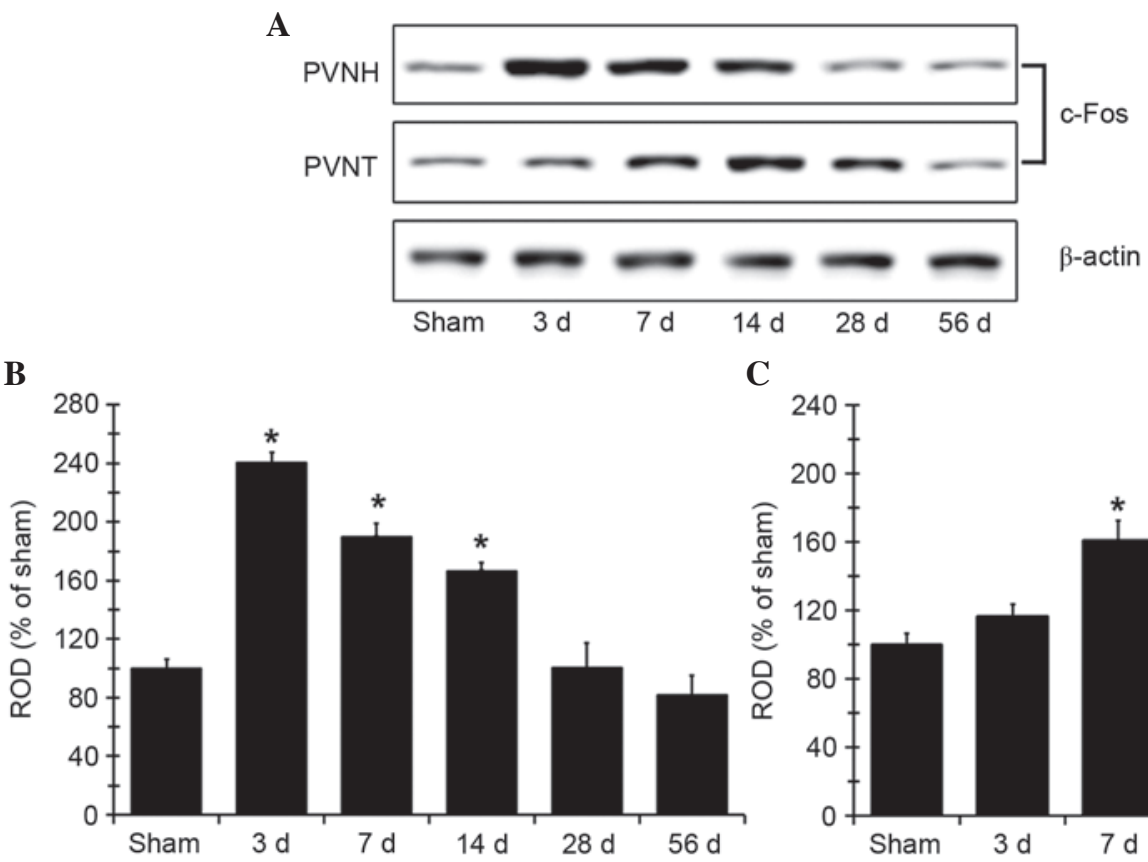

C

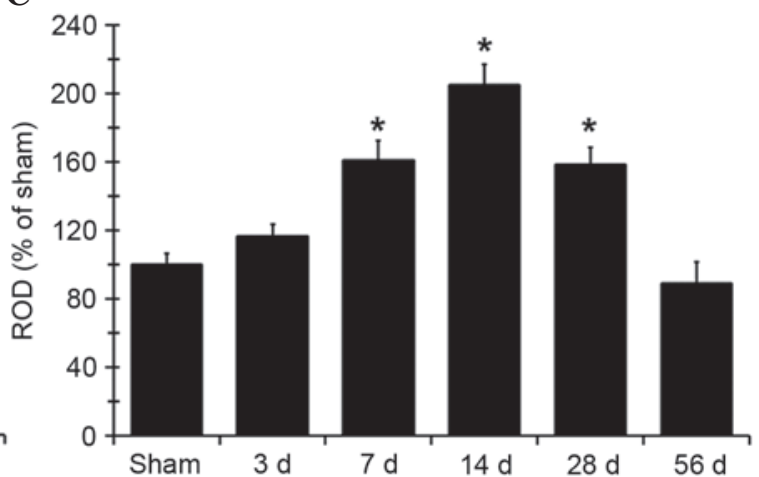

Figure 5. c-Fos expression in PVNH and PVNT. (A) Western blot analysis of c-Fos protein in the PVNH and PVNT of the sham- and MI-operated rats in sham, 3, 7, 14, 28 and 56 days after MI. c-Fos protein expression is represented as ROD of target immunoblot band to the sham band in the (B) PVNH and (C) PVNT ( $\mathrm{n}=7$ per group). ${ }^{*} \mathrm{P}<0.05$ vs. sham group. Data are presented as the mean \pm standard error. MI, myocardial infarction; PVNH, paraventricular nucleus of the hypothalamus; PVNT, paraventricular nucleus of the thalamus; c-Fos, proto-oncogene c-Fos; d, day; ROD, relative optical density.

(CNS) $(32,33)$. Additionally, it has been reported that c-Fos is useful for investigating the neuronal plasticity required for spatial memory processes (33-35). A previous study demonstrated that pain and immobilization stress increased the number of neurons exhibiting Fos-like immunoreactivity in several regions of the brain, including the PVNH (36). However, the association between c-Fos expression and neuronal activation in the CNS following MI has not been fully elucidated. It was previously reported in a rat model of the MI, that the number of $\mathrm{c}-\mathrm{Fos}^{+}$cells was increased in the hypothalamus containing PVNH (11). Additionally, changes in the number of c-Fos $^{+}$cells in the PVNH occurred at 2 and 4 weeks following MI compared with the sham group in a mouse model (20). The present study determined that the number of $\mathrm{c}-\mathrm{Fos}^{+}$cells in the PVNH peaked 3 days after MI induction, and subsequently gradually decreased and were comparable with sham controls at 56 days after MI. By contrast with previous findings $(11,20)$, the present study observed that the number of $\mathrm{c}-\mathrm{Fos}^{+}$cells in the PVNH peaked at an early time-point (3 days) after MI.

However, it was determined that the number of $\mathrm{c}-\mathrm{Fos}^{+}$cells in the PVNT was significantly increased compared with the sham group until 28 days after MI and subsequently decreased with time and were comparable with the sham group at 56 days after MI. To the best of our knowledge, the present study was the first to determine that $\mathrm{c}-$ Fos remains expressed in the PVNT to a late time-point following MI. This indicates that the increase of $\mathrm{c}^{-F_{0}}{ }^{+}$cells in the PVNT may be associated with the stress response following MI.

In conclusion, the present study did not detect MI-induced neuronal damage in the PVNH and PVNT. Whereas, MI induced different time-dependent c-Fos expression patterns in the PVNH and PVNT. This suggests that an increased number of $\mathrm{c}-\mathrm{FOS}^{+}$cells in the PVNH and PVNT may be associated with MI-induced stress. 


\section{Acknowledgements}

The present study was supported by Basic Science Research Program through the National Research Foundation of Korea funded by the Ministry of Education (grant no. NRF-2014R1 A1A2057263), the Gangwon Cardiovascular Health Research Institute and a 2014 research grant from Kangwon National University (grant no. 120140271).

\section{References}

1. Van de Werf F, Bax J, Betriu A, Blomstrom-Lundqvist C, Crea F, Falk V, Filippatos G, Fox K, Huber K, Kastrati A, et al: ESC Committee for Practice Guidelines (CPG): Management of acute myocardial infarction in patients presenting with persistent ST-segment elevation: The Task Force on the Management of ST-Segment Elevation Acute Myocardial Infarction of the European Society of Cardiology. Eur Heart J 29: 2909-2945, 2008.

2. Kim MS and Kim JJ: Heart and brain interconnection - clinical implications of changes in brain function during heart failure. Circ J 79: 942-947, 2015.

3. Hodsman GP, Kohzuki M, Howes LG, Sumithran E, Tsunoda K and Johnston CI: Neurohumoral responses to chronic myocardial infarction in rats. Circulation 78: 376-381, 1988.

4. Frahm C, Haupt $C$ and Witte OW: GABA neurons survive focal ischemic injury. Neuroscience 127: 341-346, 2004.

5. Wann BP, Boucher M, Kaloustian S, Nim S, Godbout R and Rousseau G: Apoptosis detected in the amygdala following myocardial infarction in the rat. Biol Psychiatry 59: 430-433, 2006

6. Schrier RW and Abraham WT: Hormones and hemodynamics in heart failure. N Engl J Med 341: 577-585, 1999.

7. Patel KP, Zhang PL and Krukoff TL: Alterations in brain hexokinase activity associated with heart failure in rats. Am J Physiol 265: R923-R928, 1993.

8. Sole MJ, Hussain MN and Lixfeld W: Activation of brain catecholaminergic neurons by cardiac vagal afferents during acute myocardial ischemia in the rat. Circ Res 47: 166-172, 1980.

9. Sole MJ, Hussain MN, Versteeg DH, de Kloet ER, Adams D and Lixfeld W: The identification of specific brain nuclei in which catecholamine turnover is increased by left ventricular receptors during acute myocardial infarction in the rat. Brain Res 235: 315-325, 1982.

10. Sole MJ, Versteeg DH, de Kloet ER, Hussain N and Lixfeld W: The identification of specific serotonergic nuclei inhibited by cardiac vagal afferents during acute myocardial ischemia in the rat. Brain Res 265: 55-61, 1983.

11. Patel KP, Zhang K, Kenney MJ, Weiss M and Mayhan WG: Neuronal expression of Fos protein in the hypothalamus of rats with heart failure. Brain Res 865: 27-34, 2000.

12. Hoffman GE, Smith MS and Verbalis JG: c-Fos and related immediate early gene products as markers of activity in neuroendocrine systems. Front Neuroendocrinol 14: 173-213, 1993.

13. Sagar SM, Sharp FR and Curran T: Expression of c-fos protein in brain: Metabolic mapping at the cellular level. Science 240: 1328-1331, 1988.

14. Rockhold RW, Acuff CG and Clower BR: Excitotoxic lesions of the paraventricular hypothalamus: Metabolic and cardiac effects. Neuropharmacology 29: 663-673, 1990.

15. Ahmet I, Tae HJ, Brines M, Cerami A, Lakatta EG and Talan MI: Chronic administration of small nonerythropoietic peptide sequence of erythropoietin effectively ameliorates the progression of postmyocardial infarction-dilated cardiomyopathy. J Pharmacol Exp Ther 345: 446-456, 2013.

16. Hwang IK, Yoo KY, Han TH, Lee CH, Choi JH, Yi SS, Lee SY, Ryu PD, Yoon YS and Won MH: Enhanced cell proliferation and neuroblast differentiation in the rat hippocampal dentate gyrus following myocardial infarction. Neurosci Lett 450: 275-280, 2009.

17. Lee CH, Hwang IK, Choi JH, Yoo KY, Han TH, Park OK, Lee SY, Ryu PD and Won MH: Calcium binding proteins immunoreactivity in the rat basolateral amygdala following myocardial infarction. Cell Mol Neurobiol 30: 333-338, 2010.
18. Lee CH, Park JH, Cho JH, Ahn JH, Yan BC, Lee JC, Shin MC, Cheon SH, Cho YS, Cho JH, et al: Changes and expressions of Redd1 in neurons and glial cells in the gerbil hippocampus proper following transient global cerebral ischemia. J Neurol Sci 344: 43-50, 2014.

19. Lee JC, Kim IH, Cho GS, Park JH, Ahn JH, Yan BC, Kwon HM, Kim YM, Cheon SH, Cho JH, et al: Ischemic preconditioning-induced neuroprotection against transient cerebral ischemic damage via attenuating ubiquitin aggregation. J Neurol Sci 336: 74-82, 2014.

20. Lindley TE, Doobay MF, Sharma RV and Davisson RL: Superoxide is involved in the central nervous system activation and sympathoexcitation of myocardial infarction-induced heart failure. Circ Res 94: 402-409, 2004.

21. Sun Y, Zhang JQ, Zhang J and Lamparter S: Cardiac remodeling by fibrous tissue after infarction in rats. J Lab Clin Med 135: 316-323, 2000.

22. Fletcher PJ, Pfeffer JM, Pfeffer MA and Braunwald E: Left ventricular diastolic pressure-volume relations in rats with healed myocardial infarction. Effects on systolic function. Circ Res 49: 618-626, 1981.

23. Kaloustian S, Wann BP, Bah TM, Girard SA, Apostolakis A, Ishak S, Mathieu S, Ryvlin P, Godbout R and Rousseau G: Apoptosis time course in the limbic system after myocardial infarction in the rat. Brain Res 1216: 87-91, 2008.

24. 23. Boucher M, Wann BP, Kaloustian S, Cardinal R, Godbout R and Rousseau G: Reduction of apoptosis in the amygdala by an $\mathrm{A}_{2 \mathrm{~A}}$ adenosine receptor agonist following myocardial infarction. Apoptosis 11: 1067-1074, 2006.

25. Arseneault-Bréard J, Rondeau I, Gilbert K, Girard SA, Tompkins TA, Godbout R and Rousseau G: Combination of Lactobacillus helveticus R0052 and Bifidobacterium longum R0175 reduces post-myocardial infarction depression symptoms and restores intestinal permeability in a rat model. Br J Nutr 107: 1793-1799, 2012.

26. Nillni EA: Regulation of the hypothalamic thyrotropin releasing hormone (TRH) neuron by neuronal and peripheral inputs. Front Neuroendocrinol 31: 134-156, 2010.

27. Li S and Kirouac GJ: Projections from the paraventricular nucleus of the thalamus to the forebrain, with special emphasis on the extended amygdala. J Comp Neurol 506: 263-287, 2008.

28. Fernandes GA, Perks P, Cox NK, Lightman SL, Ingram CD and Shanks N: Habituation and cross-sensitization of stress-induced hypothalamic-pituitary-adrenal activity: Effect of lesions in the paraventricular nucleus of the thalamus or bed nuclei of the stria terminalis. J Neuroendocrinol 14: 593-602, 2002.

29. Penzo MA, Robert V, Tucciarone J, De Bundel D, Wang M, Van Aelst L, Darvas M, Parada LF, Palmiter RD, He M, et al: The paraventricular thalamus controls a central amygdala fear circuit. Nature 519: 455-459, 2015.

30. Spencer SJ, Fox JC and Day TA: Thalamic paraventricular nucleus lesions facilitate central amygdala neuronal responses to acute psychological stress. Brain Res 997: 234-237, 2004.

31. Otake K, Kin K and Nakamura Y: Fos expression in afferents to the rat midline thalamus following immobilization stress. Neurosci Res 43: 269-282, 2002.

32. Radulovic J, Kammermeier J and Spiess J: Relationship between fos production and classical fear conditioning: Effects of novelty, latent inhibition, and unconditioned stimulus preexposure. J Neurosci 18: 7452-7461, 1998.

33. Tischmeyer $\mathrm{W}$ and Grimm R: Activation of immediate early genes and memory formation. Cell Mol Life Sci 55: 564-574, 1999.

34. Vanelzakker MB, Zoladz PR, Thompson VM, Park CR, Halonen JD, Spencer RL and Diamond DM: Influence of Pre-Training Predator Stress on the Expression of c-fos mRNA in the Hippocampus, Amygdala, and Striatum Following Long-Term Spatial Memory Retrieval. Front Behav Neurosci 5: 30, 2011.

35. Vann SD, Brown MW and Aggleton JP: Fos expression in the rostral thalamic nuclei and associated cortical regions in response to different spatial memory tests. Neuroscience 101: 983-991, 2000.

36. Senba E, Matsunaga K, Tohyama M and Noguchi K: Stress-induced c-fos expression in the rat brain: Activation mechanism of sympathetic pathway. Brain Res Bull 31: 329-344, 1993. 